\title{
Quantifying Itch: Measurement on the Way to Management
}

\author{
Martina L. Porter, MD; Alexa B. Kimball, MD, MPH
}

d tc tch is one of the most protean manifestations of skin disease and can take a substantial physical and emotional toll on patients. For physicians, it is a frequent-if often dreaded-patient concern with a rising incidence. Lack of specific itch therapies as well as associations with multiple dermatologic conditions, including xerosis, psoriasis, atopic dermatitis, cutaneous lymphoma, contact dermatitis, and internal malignancies, make management of these itchy patients challenging and deserving of our attention. Studies evaluating patients with chronic pruritus identified a considerable impact on healthrelated quality of life, including development of depression, inability to perform activities of daily living, and sleep difficulties. ${ }^{1}$

\section{How to Classify Itching}

Itch, or pruritus, originally was defined as an unpleasant sensation that provokes the desire to scratch, ${ }^{2}$ but this definition likely limits our ability to assess itch. The urge to scratch the skin to relieve the itch is sometimes a reflex of the muscles triggered by the spinal cord that can be either conscious or unconscious. If 2 patients present with itch, does the patient with more excoriated skin experience more severe itch? Conversely, does the patient who scratches less have an equivalent decrease in itch severity? Although it is tempting to quantify itch through physical signs such as excoriations, it ultimately is a subjective symptom that is difficult to assess.

Pain is another complex subjective symptom but is one that has been better studied. A previous intensity theory postulated that itch is a form of pain: low-intensity noxious stimuli are perceived as itch, while high-intensity stimuli are perceived as pain. Over time, our understanding of itch evolved, and it became clear that a specific neuronal pathway for itch also exists. ${ }^{3}$ However, the pathophysiology of itch and pain remain intertwined. Scratching may elicit pain, providing a change in sensation that replaces the itch, whereas opioid analgesics suppress pain but may worsen the itch.

We are gaining a better understanding of the biology and classification of itch, which will hopefully enable the development of new measures to accurately assess itch. Four main categories of itch currently exist: neurogenic, psychogenic, neuropathic, and pruritoceptive. ${ }^{4}$ Patients may have one or multiple types of itch, which can be differentiated clinically and biochemically. Neurogenic (also known as systemic) itch is transmitted via the central nervous system with possible involvement of itch-specific neurons in the spinal cord and encompasses itch associated with pruritus from other organ systems. As the term implies, psychogenic itch is associated with psychiatric disorders. Neuropathic itch is generated from the inappropriate firing of peripheral or central sensory neurons in the absence of pruritogenic stimuli, which can be seen in notalgia paresthetica, brachioradial pruritus, and postherpetic neuralgia. Pruritoceptive itch most commonly is encountered in dermatology and is associated with skin inflammation or other dermatoses. ${ }^{4}$

\section{How to Assess Itch Quantitatively}

There currently are 2 major questions about quantitative assessments of itch. First, how do we measure itch

\footnotetext{
From Harvard Medical School, Boston, Massachusetts, and the Clinical Laboratory for Epidemiology and Applied Research in Skin (CLEARS), Department of Dermatology, Beth Israel Deaconess Medical Center, Boston.

Dr. Porter is a consultant and/or investigator for AbbVie Inc, Bristol Meyers Squibb, Eli Lilly and Company, Janssen Pharmaceuticals, Novartis, Pfizer, and UCB. Dr. Kimball is a consultant and investigator for AbbVie Inc, Bristol Myers Squib, Janssen Pharmaceuticals, Novartis, Pfizer, Regeneron Pharmaceuticals, and UCB. Dr. Kimball also receives fellowship funding from AbbVie Inc and Janssen Pharmaceuticals. She currently is on the Board of Directors for Almirall and previously served on the Board of Directors for the Hidradenitis Suppurativa Foundation. She also served on the Board of Directors for and as past president of the International Psoriasis Council. Correspondence: Alexa Kimball, MD, MPH, Clinical Laboratory for Epidemiology and Applied Research in Skin (CLEARS), Department of Dermatology, Beth Israel Deaconess Medical Center, 330 Brookline Ave, Boston, MA 02115 (abkimbal@bidmc.harvard.edu). doi:10.12788/cutis.0228
} 
in studies that are designed to relieve a different skin disease that is associated with itch? Most clinical trials investigating therapeutic options for atopic dermatitis and psoriasis now include itch assessment and improvement as a secondary outcome. Second, how do we measure itch in studies that are designed with relief of itch as the primary end point? Both of these scenarios require a fundamental set of decisions. Itch clearly is a subjective experience, but it also is one that can be local, regional, generalized, or transitory. Just as with pain, an individual can be distracted from their itch to some extent and consequently experience it more acutely when there are fewer stimuli in their environment. Classically, patients will report that itching is worse at night, preventing them from sleeping. Sleep disruption previously has been demonstrated. ${ }^{5}$ Of course, the environment also can exacerbate itch, as dry air and in some cases humidity can flare the sensation.

Fundamentally, therefore, the questions that are asked to assess itch are incredibly relevant, and there is a matrix of possible avenues of inquiry. Should you measure the peak itch in one area or the peak itch overall? Is the duration, the frequency, or the persistence of the itching most relevant? What is the correct time frame in which to do an assessment: the last 24 hours, the last 48 hours, or the last week? Because these parameters have been so challenging, most investigators have used a visual analog scale, similar to what is used to assess pain, at a 24-hour interval to decrease recall bias. The most commonly employed tool is the itch numeric rating scale (NRS), which asks patients to rate their symptoms on a scale of 0 (no itch) to 10 (worst imaginable itch). Although the psychometric properties of the itch NRS have been validated, debate still exists as to whether the itch NRS is best administered at a specific time of day or if it should be updated to evaluate peak pruritus scores explicitly. Regardless, implementing these scales often is time consuming and burdensome in the clinical trial setting, as participants are asked to complete daily diaries at the same time each day using either paper forms or electronic tablets.

Once scores are collected, we then need to quantitate a meaningful difference in itch. For pain, there has been some acceptance of a $30 \%$ difference, or a 2 -point reduction, as being clinically meaningful; however, there was substantial debate at the time of the approval of ixekizumab as to whether that was a similarly appropriate threshold for itch. Using data from ixekizumab phase 2 and phase 3 trials, a 4-point reduction in itch NRS was found to be optimal for evaluating clinically significant changes in moderate to severe psoriasis. ${ }^{6}$ A more recent study of the validity of the itch NRS in prurigo nodularis suggested a 1-point change was correlated with minimal clinical improvement. ${ }^{7}$ Thus, the interesting question of how assessment of itch varies across clinical trials and disease states needs to be raised. Psoriasis classically has been thought of as not particularly itchy, and atopic dermatitis and prurigo nodularis have been regarded as extraordinarily itchy, yet one study comparing baseline itch scores in psoriasis and atopic dermatitis suggested that the experience actually is somewhat similar. ${ }^{8}$

\section{Final Thoughts}

The subjective nature of itch makes NRSs our best option at this time, but the best disease severity assessment tools are objective, sensitive, and generalizable. Unfortunately, we do not have such tools available to us yet, but technology—smart devices to monitor nocturnal scratching and machine learning algorithms that use electromagnetic impact to capture motion associated with itching and scratching - may offer new objective measures for itch that can be used to further validate the current itch NRS. Even if these technology-based approaches become the standard of measurement, they will certainly help us understand what we are measuring. And even better, the focus on how to develop meaningful end points around the improvement of itch will likely lead us to measure it more and drive the development of therapeutics that address the effect and consequences of this pernicious problem.

\section{REFERENCES}

1. Kini SP, DeLong LK, Veledar E, et al. The impact of pruritus on quality of life: the skin equivalent of pain. Arch Dermatol. 2011;147:11531156. doi:10.1001/archdermatol.2011.178

2. Savin JA. How should we define itching? I Am Acad Dermatol. 1998;39(2 pt 1):268-269. doi:10.1016/s0190-9622(98)70087-8

3. Ikoma A, Rukwied R, Ständer S, et al. Neurophysiology of pruritus: interaction of itch and pain. Arch Dermatol. 2003; 139:1475-1478. doi:10.1001/archderm.139.11.1475

4. Garibyan L, Rheingold CG, Lerner EA. Understanding the pathophysiology of itch. Dermatol Ther. 2013;26:84-91. doi:10.1111/ dth.12025

5. Kimball AB, Luger T, Gottlieb A, et al. Impact of ixekizumab on psoriasis itch severity and other psoriasis symptoms: results from 3 phase III psoriasis clinical trials. J Am Acad Dermatol. 2016;75:1156-1161. doi:10.1016/j.jaad.2016.07.034

6. Kimball AB, Naegeli AN, Edson-Heredia E, et al. Psychometric properties of the Itch Numeric Rating Scale in patients with moderate-tosevere plaque psoriasis. Br J Dermatol. 2016;175:157-162. doi:10.1111/ bjd.14464

7. Kimel M, Zeidler C, Kwon P, et al. validation of psychometric properties of the itch numeric rating scale for pruritus associated with prurigo nodularis: a secondary analysis of a randomized clinical trial. JAMA Dermatol. 2020;156:1354-1358. doi:10.1001/jamadermatol.2020.3071

8. Shahwan KT, Kimball AB. Itch intensity in moderate-to-severe plaque psoriasis versus atopic dermatitis: a meta-analysis. J Am Acad Dermatol. 2017;76:1198.el-1200.e1. doi:10.1016/j.jaad.2017.02.002

9. Smith MP, Ly K, Thibodeaux Q, et al. Emerging methods to objectively assess pruritus in atopic dermatitis. Dermatol Ther (Heidelb). 2019;9:407420. doi:10.1007/s13555-019-0312-3 